\title{
PERTUZUMAB FOR THE TREATMENT OF PATIENTS WITH PREVIOUSLY UNTREATED HER2-POSITIVE METASTATIC BREAST CANCER
}

\author{
M.B. Smith, J. Reardon, and E.M. Olson \\ James Cancer Hospital and Solove Research Institute, The Ohio State University, Columbus, \\ Ohio, USA
}

\section{SUMMARY}

Pertuzumab is a humanized monoclonal antibody directed at the dimerization domain of the receptor tyrosine-protein kinase erbB-2 (HER2) receptor. It possesses a unique and complimentary mechanism of action compared to trastuzumab, which has historically been the cornerstone of therapy for HER2-amplified breast cancer. Clinical trials demonstrate improved outcomes, with minimal increases in toxicity with the addition of pertuzumab to trastuzumab in patients with HER2-positive metastatic breast cancer, indicating the advantage of dual HER2 receptor blockade. Pertuzumab is approved as first-line therapy in combination with trastuzumab and docetaxel for HER2-positive metastatic breast cancer, with future opportunities to investigate its efficacy in other stages of breast cancer, as well as in the treatment of other malignancies.

\section{Keywords}

Humanized monoclonal antibody; HER2 receptor; Metastatic breast cancer; Pertuzumab

\section{INTRODUCTION}

Molecular alterations in the human receptor tyrosine-protein kinase erbB family of receptors is a common event found in multiple epithelial tumor types. Genetic modifications of these transmembrane receptors, which include epidermal growth factor receptor (EGFR, receptor tyrosine-protein kinase erbB-1), receptor tyrosine-protein kinase erbB-2 (HER2), receptor tyrosine-protein kinase erbB-3 (HER3) and receptor tyrosine-protein kinase erbB-4 (HER4), are well documented in breast, lung, prostate, colorectal and ovarian cancers (1). The presence of these cellular proteins is associated with tumor aggressiveness and translates into a negative clinical prognostic indicator in several solid tumor malignancies (2). As a result, this family of receptors is a common molecular target for oncological drug discovery, and results to date suggest that inhibition of HER signaling leads to effective anticancer treatment.

The malignant site in which blockade of the HER family of receptors has led to the greatest impact is breast cancer; approximately $15-25 \%$ of all breast tumors have amplification or overexpression of the transmembrane glycoprotein HER2. Aberrant HER2 activity plays a

Copyright () 2012 Prous Science, S.A.U. or its Licensors. All rights reserved

Correspondence: Erin M. Olson, MD, James Cancer Hospital and Solove Research Institute, The Ohio State University, B407 Starling Loving Hall, Columbus, OH 43212, USA., erin.olson@ osumc.edu.

DISCLOSURES

The authors state no conflicts of interest. 
significant role in tumor initiation and aggressive cellular behavior through the activation of a variety of molecular pathways (3,4). A number of anti-HER2 treatment options are approved for use in patients with HER2-positive breast cancer. Trastuzumab, a monoclonal antibody (MAb) created to inhibit the extracellular domain IV of HER2, has dramatically improved clinical outcomes in both treatment-naive and treatment-refractory patients. Lapatinib, a small-molecule tyrosine kinase inhibitor that blocks the intracellular domain of HER2, is also used in patients with advanced or metastatic breast cancer. Prior to the discovery of HER2-directed agents, dependency on the HER2 pathway was an independent predictor of reduced disease-free and overall survival (3); in the modern era, patients treated with HER2-directed therapy demonstrate similar survival as patients with HER2-negative disease (5).

Pertuzumab is the newest agent targeting HER2, and it was recently approved as part of combination therapy for use in patients with HER2-amplified metastatic breast cancer without prior treatment for metastatic disease with anti-HER2 agents or chemotherapy. Because pertuzumab acts on a separate domain (extracellular domain II) of the HER2 receptor from trastuzumab, it's mechanism of action is distinct and complimentary to traditional trastuzumab-based therapy (6). This review describes the pharmacology, safety, efficacy and other clinical considerations associated with pertuzumab.

\section{PRECLINICAL PHARMACOLOGY}

Dimerization of HER2 with itself (homodimerization) or with other members of the HER family (heterodimerization) leads to signaling via the mitogen-activated protein kinase (MAPK) and phosphatidylinositol 3-kinase (PI3K) pathways, resulting in tumor cell proliferation and cell survival. The most potent is the HER2:HER3 heterodimer, as high levels of this protein complex cause robust pro-oncogenic signaling and resistance to trastuzumab-based therapy (7). In preclinical studies, the recombinant humanized MAb 2C4, now named pertuzumab, blocked HER2:HER3 heterodimer formation in breast cancer cell lines that express both HER2 and HER3 (8). Pertuzumab binds to the dimerization arm (domain II) of HER2 with high affinity, sterically hindering HER2 signaling by inhibiting interactions with HER3. Similar to trastuzumab, pertuzumab can also cause antibodydependent cell-mediated cytotoxicity (ADCC) by engaging immune effector cells via their receptors for the IgG immunoglobulin (9), leading to cancer cell death (10).

Importantly, pertuzumab and trastuzumab attach to discrete domains of the HER2 receptor, leading to complimentary blockade of HER2-associated growth (Fig. 1). Nahta et al. (11) tested the survival of human breast ductal carcinoma BT-474 (HER2-overexpressing) cell lines treated with pertuzumab, trastuzumab or a combination of both. The investigators found that combining both HER2 antibodies synergistically inhibited BT-474 cell survival by $60 \%$ compared to limited activity of either agent alone. Scheuer et al. (10) confirmed these initial efficacy studies by testing the activity of pertuzumab in combination with trastuzumab in breast cancer xeno-graph models. Six of 10 mice treated with dual HER2 blockade with the antibody combination had complete tumor remission. Additionally, tumor mass reduction was seen in murine tumors that progressed on single-agent trastuzumab that were then treated with pertuzumab and trastuzumab.

\section{PHARMACOKINETICS AND METABOLISM}

Adams et al. (12) reported preclinical pharmacokinetic (PK) parameters for pertuzumab in mice, rats and cynomolgus monkeys that supported the dosing regimens used during clinical development. Based on single-dose studies in male CD-1 mice and male Sprague Dawley rats, as well as single-dose and multiple-dose studies in male and female cynomolgus monkeys, pertuzumab was found to display biphasic disposition, fitting into a two- 
compartment PK model. The volume of distribution $\left(\mathrm{V}_{\mathrm{d}}\right)$ was $27-58 \mathrm{~mL} / \mathrm{kg}$, with the $\mathrm{V}_{\mathrm{d}}$ of the central compartment approximating the serum volume in all models. The distribution phase was less than 24 hours. The terminal elimination half-life was approximately 10 days. Based on PK simulations from these results, dosing of pertuzumab 5-15 mg/kg every 3 weeks would achieve a target trough concentration of $20 \mu \mathrm{g} / \mathrm{mL}$.

A phase I trial evaluating the safety and tolerability of escalating doses of pertuzumab in 21 U.S. patients with locally advanced, recurrent or metastatic solid tumors was undertaken by Agus et al. (13). Pertuzumab doses ranged from $0.5 \mathrm{mg} / \mathrm{kg}$ to $15 \mathrm{mg} / \mathrm{kg}(0.5,2.0,5.0,10.0$ and $15.0 \mathrm{mg} / \mathrm{kg}$ ) intravenously every 3 weeks. At all dose levels except for $0.5 \mathrm{mg} / \mathrm{kg}$, there were no differences in clearance (mean $3.42 \pm 1.20 \mathrm{~mL} / \mathrm{day} / \mathrm{kg}$ ), $\mathrm{V}_{\mathrm{d}}$ of the central compartment (mean $40.6 \pm 6.6 \mathrm{~mL} / \mathrm{kg}), \mathrm{V}_{\mathrm{d}}$ at steady state $(80.0 \pm 28.2 \mathrm{~mL} / \mathrm{kg})$ and elimination half-life (mean $18.9 \pm 8$ days). Pertuzumab demonstrated similar pharmacokinetics to other $\operatorname{IgG}_{1}$ monoclonal antibodies $(12,13)$. No maximum tolerated dose was observed. The doses of. 10 and $15 \mathrm{mg} / \mathrm{kg}$ resulted in trough concentrations $>20 \mu \mathrm{g} / \mathrm{mL}$. The authors note that this was favorable, as nonclinical models showed that for pertuzumab steady-state trough concentrations between 5 and $25 \mu \mathrm{g} / \mathrm{mL},>80 \%$ suppression of tumor growth is achieved.

A second phase I dose-escalation PK trial was conducted in 18 Japanese patients by Yamamoto et al. (16). Pertuzumab doses ranged from 5 to $25 \mathrm{mg} / \mathrm{kg}(5,10,15,20$ and 25 $\mathrm{mg} / \mathrm{kg}$ ) intravenously every 3 weeks. As with the previous study, there were no differences in clearance $(4.30 \pm 1.72 \mathrm{~mL} / \mathrm{d} / \mathrm{kg}), \mathrm{V}_{\mathrm{d}}$ of the central compartment, $\mathrm{V}_{\mathrm{d}}$ at steady state $(92.4$ $\pm 15.2 \mathrm{~mL} / \mathrm{kg}$ ) and terminal half-life (17.3 days) between dosing cohorts. Pertuzumab displayed linear PK with increasing doses, as shown by proportional increases in $\mathrm{C}_{\max }$ and AUC ( $r=0.914$ and $r=0.808$, respectively). Similar to the previous study, no maximum tolerated dose was observed. These PK findings suggest no major difference between patient populations, and both phase I trials support the use of dosing every 3 weeks.

$\mathrm{Ng}$ et al. (17) developed a two-compartment linear population PK model for pertuzumab based on the PK results of 1,458 pertuzumab serum concentrations from 153 patients in a phase I and two phase II trials. Using a bootstrapping resampling technique, PK results were simulated for 1,000 pertuzumab subjects receiving treatment every 3 weeks with either fixed dosing ( $840 \mathrm{mg} \times 1$, followed by $420 \mathrm{mg})$, weight-based dosing $(12.2 \mathrm{mg} / \mathrm{kg} \times 1$, followed by $6.1 \mathrm{mg} / \mathrm{kg}$ ) or body surface area (BSA)-based dosing $\left(485 \mathrm{mg} / \mathrm{m}^{2} \times 1\right.$, followed by 242.5 $\mathrm{mg} / \mathrm{m}^{2}$ ). The clearance of pertuzumab was found to be $0.214 \mathrm{~L} /$ day with $a V_{d}$ of the central compartment of $40 \mathrm{~mL} / \mathrm{kg}$. All dosing regimens consistently kept serum trough concentrations greater than the target of $20 \mu \mathrm{g} / \mathrm{mL}$ more than $90 \%$ of the time, with weightbased and BSA-based dosing trough concentrations lower than fixed dosing by $6.17 \%$ and $5.76 \%$, respectively. The percentage of patients with trough concentrations lower than 20 $\mu \mathrm{g} / \mathrm{mL}$ was similar in patients weighing in either $\leq 10^{\text {th }}$ or $\geq 90^{\text {th }}$ percentile. Although it was found that serum albumin and weight affected clearance, and that $V_{d}$ of the central compartment was influenced by BSA, it was concluded that weight-based and BSA-based dosing did not improve steady-state exposure to pertuzumab. Therefore, a fixed dosing regimen of pertuzumab every 3 weeks was recommended.

The metabolism of pertuzumab has not been formally studied. It was reported by Mariani et al. (18) that IgG metabolism occurs prominently in the liver, and to a lesser extent in the kidneys and gastrointestinal tract. Catabolic degradation in the liver occurs in the reticuloendothelial system and endothelial cells (19). Pertuzumab does not appear to undergo metabolism by the cytochrome P450 enzyme family. 


\section{SAFETY}

Single-agent pertuzumab is generally very well tolerated. In two phase I studies, the most commonly reported adverse events (AEs) were asthenia, nausea, vomiting, diarrhea and rash, with the majority being NCI-CTC grade 1 or grade $2(13,16)$. An open-label phase II study by Glanni et al. (20) compared two different fixed-dose regimens (420 mg vs. 1050 mg every 3 weeks) of single-agent pertuzumab in HER2-negative metastatic breast cancer patients. Again, the most common AEs were grade 1 and 2 diarrhea (43.9-45.9\%), nausea (24.4-27\%), fatigue (19.5-24.3\%), rash (19.5-21.6\%) and vomiting (12.2-16.2\%). The only reported grade $3 \mathrm{AEs}$ were diarrhea $(5.4-7.3 \%)$, fatigue $(<3 \%)$ and vomiting $(<3 \%)$. In another phase II study, Cortés et al. (21) evaluated pertuzumab monotherapy in HER2positive advanced breast cancer, and toxicity results were similar to those previously reported. When pertuzumab was used in combination with trastuzumab for the treatment of advanced or metastatic breast cancer, there were no significant changes to the toxicity profiles described above $(21,22)$.

In the Large phase III CLEOPATRA trial reported by Baselga et al. (23), either pertuzumab or placebo was added to docetaxel and trastuzumab for first-line treatment of HER2-positive metastatic breast cancer. Toxicity profiles between the pertuzumab and placebo groups were not dramatically different. For all grades of AEs, diarrhea, rash, mucosal inflammation, febrile neutropenia and dry skin were more common in the pertuzumab group than in the placebo group. When comparing grade 3 or higher toxicity, the pertuzumab group had increased rates of neutropenia ( $48.9 \%$ vs. $45.8 \%)$, febrile neutropenia ( $13.8 \%$ vs. $7.6 \%)$ and diarrhea ( $7.9 \%$ vs.5.0\%) compared to control. Deaths related to adverse effects of the treatment were $2.5 \%$ and $2.0 \%$ for the placebo and pertuzumab groups, respectively, with infection being the primary cause.

The phase II NeoSphere trial by Gianni et al. (24) also looked at pertuzumab in combination with trastuzumab and docetaxel, although the treatment was in the neoadjuvant setting for locally advanced, inflammatory or early-stage HER2-positive breast cancer patients. In this trial, patients were randomized to one of four groups: trastuzumab plus docetaxel (group A), pertuzumab plus trastuzumab plus docetaxel (group B), pertuzumab plus trastuzumab (group C), or pertuzumab plus docetaxel (group D). The most common AEs were alopecia, neutropenia, diarrhea, nausea, fatigue, rash and mucosal inflammation. The toxicity rates between chemotherapy-containing groups (A, B and D) were similar. In the group in which no chemotherapy was given (group C), alopecia, neutropenia and mucosal inflammation occurred in $\leq 3 \%$ of patients. Febrile neutropenia occurred in $7-8 \%$ of patients in groups A, $\mathrm{B}$ and $\mathrm{D}$, compared to no events in group $\mathrm{C}$. These results suggest that pertuzumab is generally well tolerated, and that most of the AEs experienced in this trial were related to the cytotoxic agent docetaxel.

Patients receiving pertuzumab should be educated on the risk of embryofetal toxicity. Pertuzumab was administered to 36 pregnant cynomolgus monkeys during the period of fetal organogenesis (gestation days 20 to 50 (25). By gestation day 70, abortion or embryofetal death was seen in 55\% of the cynomolgus monkeys. Cesarean section was planned on day 100 for the remaining animals Oligohydramnios was accompanied by delayed development of the fetal kidneys, as well as some external, visceral and skeletal abnormalities.

\section{Cardiotoxicity risk}

It is well known that trastuzumab induces some cardiotoxicity. Since both pertuzumab and trastuzumab act on the HER2 receptor, albeit at different domains, there is a baseline concern that pertuzumab may cause similar effects on the heart. During the phase I 
pertuzumab trials, patients with a baseline left ventricular ejection fraction (LVEF) $<50 \%$ were not studied $(13,16)$. In these trials, one patient suffered a myocardial infarction possibly leading to left ventricular failure, and three patients experienced asymptomatic decreases in baseline LVEF ranging from 5\% to $14 \%$ (13). In the phase II trials evaluating pertuzumab monotherapy, 7-10\% of patients experienced drops in LVEF $\geq 10 \%$ accompanied by LVEF $<50 \%(20,21)$. In a phase II study by Baselga et al. (22), in which patients received treatment with both trastuzumab and pertuzumab, $4.5 \%$ of patients experienced drops in LVEF $\geq 10 \%$ accompanied by LVEF $<50 \%$. Pertuzumab was also investigated in combination with trastuzumab using stringent criteria for cardiotoxicity evaluation in a phase II study by Portera et al. (26). This trial was stopped early after 6 of the first 11 patients experienced a reduction in LVEF $<55 \%(\mathrm{n}=3,2$ and 1 for grades 1,2 and 3 , respectively), though only 1 patient had symptomatic congestive heart failure. The authors noted a concern for safety and the need for close monitoring in future studies utilizing the combination of pertuzumab and trastuzumab.

Surprisingly, in the CLEOPATRA trial by Baselga et al. (23), patients in the placebo group demonstrated more left ventricular systolic dysfunction than those administered pertuzumab (all grades: $8.3 \%$ vs. $4.4 \%$; grades 3-4: $2.8 \%$ vs. $1.2 \%$, respectively). LVEF assessments showed $6.6 \%$ in the placebo group compared to $3.8 \%$ in the pertuzumab group had a $\geq 10 \%$ decline from baseline accompanied by LVEF $<50 \%$. Patients were excluded from the trial if baseline $\mathrm{LVEF}<50 \%,>360 \mathrm{mg} / \mathrm{m}^{2}$ of doxorubicin (or anthracycline equivalent) or LVEF $<50 \%$ during or after prior trastuzumab therapy.

There was no significant increased risk in LVEF dysfunction seen when pertuzumab was added to trastuzumab in the neoadjuvant trial NeoSphere by Gianni et al. (24). The prevalence of LVEF, which declined $210-15 \%$ from baseline accompanied by LVEF < $50 \%$ in patients receiving trastuzumab plus docetaxel, pertuzumab plus trastuzumab plus docetaxel, pertuzumab plus trastuzumab or pertuzumab plus docetaxel, was $0.9 \%, 2.8 \%$, $0.9 \%$ and $1.1 \%$, respectively. All but one patient had their LVEF $>50 \%$ and a decrease of $<$ $10 \%$ by the fourth cycle of treatment. This patient, who had a significant cardiac medical history, discontinued treatment due to heart failure. The concern for pertuzumab to cause cardiotoxicity, as well as increase in the risk of cardiotoxicity when added to trastuzumab therapy, was addressed throughout the pertuzumab clinical trials. During phase I and II monotherapy studies, pertuzumab did show modest cardiotoxicity similar to that of trastuzumab. There was some initial concern for excessive cardiotoxicity based on the early phase II results of Portera et al. (26). However, larger phase III trials utilizing appropriate monitoring showed that when used in combination with trastuzumab, pertuzumab does not appear to increase the risk of cardiotoxicity compared to trastuzumab alone.

\section{CLINICAL STUDIES}

Due to the complementary mechanisms of action of pertuzumab and trastuzumab in the inhibition of HER2 signaling, research to date in the setting of HER2-positive breast cancer has primarily investigated the use of both agents in combination. The single-arm phase II trial by Baselga et al. (22) assessed the efficacy and safety profile of the combination in 66 HER2-amplifed patients with evidence of progression on trastuzumab-based therapy. Trastuzumab was given using either a weekly regimen $(4 \mathrm{mg} / \mathrm{kg}$ loading dose followed by 2 $\mathrm{mg} / \mathrm{kg}$ every week) or administered on an every-3-week basis $(8 \mathrm{mg} / \mathrm{kg}$ loading dose followed by $6 \mathrm{mg} / \mathrm{kg}$ every 3 weeks). The dosing schedule of pertuzumab was an 840-mg loading dose followed by $420 \mathrm{mg}$ every 3 weeks. The objective response (OR) rate across the study population was $24.2 \%$, and the clinical benefit rate, defined to include both patients with an OR and patients with stable disease for greater than 6 months, was 50\%. Median progression-free survival (PFS) was 5.5 months. These results compared favorably

Drugs Today (Barc). Author manuscript; available in PMC 2013 September 30. 
to other treatment combinations in a trastuzumab-refractory HER2-positive population, including combinations such as trastuzumab plus lapatinib (27), trastuzumab plus capecitabine (28) and lapatinib plus capecitabine (29).

Following up on the clinical benefit seen with the combination of pertuzumab and trastuzumab, another phase II study by Cortés et al. (21) was conducted to determine if the activity was secondary to the combination of the anti-HER 2 agents, or whether pertuzumab alone was the agent responsible for the increase in efficacy. A cohort of 29 patients with HER2-positive breast cancer with previous treatment on a trastuzumab-containing regimen received pertuzumab as an 840-mg loading dose followed by $420 \mathrm{mg}$ every 3 weeks. At the time of tumor growth, 17 patients were reintroduced to trastuzumab at either weekly or every-3-week dosing, while continuing pertuzumab at the same dose. All 29 patients enrolled in the study progressed during pertuzumab monotherapy, with an OR rate and clinical benefit rate of $3.4 \%$ and $10.3 \%$, respectively. After the reintroduction of trastuzumab in combination therapy with pertuzumab, the OR rate and clinical benefit rate increased to $17.6 \%$ and $41.2 \%$, respectively. PFS was also longer with combination therapy (17.4 weeks) when compared with pertuzumab monotherapy (7.1 weeks). Although the sample size was small, pertuzumab monotherapy appears to have modest single-agent activity, and improved antitumor activity may be achieved when HER2 is maximally inhibited by dual HER2 blockade.

The effects of pertuzumab have also been studied in conjunction with chemotherapy. In the phase II study by Gianni et al. (24) evaluating treatment-naive women with early-stage HER2-overexpresstng breast cancer, patients received four neoadjuvant cycles of varying combinations of trastuzumab ( $8 \mathrm{mg} / \mathrm{kg}$ loading dose followed by $6 \mathrm{mg} / \mathrm{kg}$ every 3 weeks), pertuzumab (840-mg loading dose followed by $420 \mathrm{mg}$ every 3 weeks) and docetaxel (75 $\mathrm{mg} / \mathrm{m}^{2}$, escalating, if tolerated, to $100 \mathrm{mg} / \mathrm{m}^{2}$ every 3 weeks). A total of 417 eligible patients were randomly assigned to 1 of 4 treatment groups: trastuzumab plus docetaxel, pertuzumab and trastuzumab plus docetaxel, pertuzumab and trastuzumab or pertuzumab plus docetaxel. Patients who received pertuzumab and trastuzumab plus docetaxel had a significantly improved pathological complete response rate $(45.8 \%)$ versus trastuzumab plus docetaxel (29.0\%; $P=0.0141)$, pertuzumab plus docetaxel $(24.0 \% ; P=0.003)$ and pertuzumab and trastuzumab $(16.8 \% ; P<0.001)$. Similar results in combination with docetaxel were demonstrated in a first-line phase III study evaluating patients with metastatic disease. In the landmark CLEOPATRA trial by Baselga et al. (23), investigators assessed the efficacy of the addition of pertuzumab to the treatment combination of trastuzumab plus docetaxel as first-line therapy. Patients were randomly assigned to receive either placebo plus trastuzumab plus docetaxel or pertuzumab plus trastuzumab plus docetaxel, and 808 patients were included in the study. Pertuzumab was given as an 840-mg loading dose, followed by $420 \mathrm{mg}$ every 3 weeks, and trastuzumab was given as an $8 \mathrm{mg} / \mathrm{kg}$ loading dose, followed by $6 \mathrm{mg} / \mathrm{kg}$ every 3 weeks. The initial dose of docetaxel was $75 \mathrm{mg} / \mathrm{m}^{2}$, which could be increased to $100 \mathrm{mg} / \mathrm{m}^{2}$ if tolerated. The median PFS was improved with the addition of pertuzumab to trastuzumab and docetaxel, with 18.5 months in the pertuzumab group versus 12.4 months in the placebo group $(P<0.001)$. Preliminary data showed a strong trend toward an overall survival benefit with the addition of pertuzumab to trastuzumab and docetaxel, with fewer deaths in the pertuzumab group (17.2\% vs. $23.6 \%)$. This difference in survival did not reach statistical significance (hazard ratio [HR]: 0.64; $P=0.005$ ), although the data were not yet fully mature, reaching only $43 \%$ of that specified for the final analysis.

Given these dramatic improvements in patient outcome, the data from the CLEOPATRA trial led to accelerated FDA approval for pertuzumab as first-line therapy in combination with trastuzumab and docetaxel for metastatic HER2-amplified breast cancer. Pertuzumab has been tested in other arenas, including HER2-negative breast cancer, with limited 
efficacy (20). Ongoing clinical trials evaluating the antitumor activity of pertuzumab are in progress and include multiple drug combinations in breast and non-breast primary malignancies (Table I).

\section{DRUG INTERACTIONS}

Studies evaluating possible drug-drug interactions with pertuzumab are limited. Scheuer et al. (10) showed that trastuzumab does not inhibit pertuzumab binding to HER2 in vivo. A phase Ib trial by Attard et al. (30) demonstrated no difference in the PK profile of pertuzumab or docetaxel when used in combination as compared to each agent alone. In a phase I study by Albanell et al. (31), pertuzumab was administered simultaneously with capecitabine without alteration in either medication's PK or toxicity profile. When pertuzumab was studied in combination with the investigational antibody-drug conjugate trastuzumab emtanslne (T-DM1) by Lu et al. (32), it was concluded that the risk of therapeutic drug-protein interactions between pertuzumab and T-DM1 was low. Pertuzumab may be administered safely without drug interactions in combination with trastuzumab and docetaxel, the combination for which it has been granted FDA approval (23). Therefore, until formal drug-drug interactions are identified, pertuzumab appears to have minimal effects on other medications, While other medications also have minimal effects on Pertuzumab.

\section{CONCLUSION}

Pertuzumab was approved by the FDA in June 2012 for the treatment of HER2-positive metastatic breast cancer Combination with trastuzumab and docetaxel in patients who have not received prior anti-HER2 therapy or chemotherapy to treat metastatic disease. The improvement in clinical outcomes with the addition of Pertuzumab to trastuzumab is seen as a result of its Complimentary mechanism of action to trastuzumab, resulting in maximal blockade of the HER2 oncogenic pathway. Combination studies of pertuzumab with trastuzumab demonstrate the regimen's superior efficacy over trastuzumab alone, with only a minimal increase in observed toxicities. Future trials will investigate pertuzumab's role in other stages of breast cancer, including its use in the neoadjuvant and adjuvant settings. Clinical trials investigating the use of pertuzumab in the treatment of other solid tumors, including gastric, neuroendocrine and colorectal cancers, are ongoing.

\section{References}

1. Aaronson SA. Growth factors and cancer. Science. 1991; 254(5035):1146-53. [PubMed: 1659742]

2. Mendelsohn J, Baselga J. The EGF receptor family as targets for cancer therapy. Oncogene. 2000; 19(56):6550-65. [PubMed: 11426640]

3. Slamon DJ, Clark GM, Wong SG, Levin WJ, Ullrich A, McGuire WL. Human breast cancer: correlation of relapse and survival with amplification of the HER-2/neu oncogene. Science. 1987; 235(4785):177-82. [PubMed: 3798106]

4. Yarden Y. The EGFR family and its ligands in human cancer: signaling mechanisms and therapeutic opportunities. Eur J Cancer. 2001; 37(Suppl 4):S3-8. [PubMed: 11597398]

5. Dawood S, Broglio K, Buzdar AU, Hortobagyi GN, Giordano SH. Prognosis of women with metastatic breast cancer by HER2 status and trastuzumab treatment: an institutional-based review. J Clin Oncol. 2010; 28(1):92-8. [PubMed: 19933921]

6. Franklin MC, Carey KD, Vaidos FF, Leahy DJ, de Vos AM, Sliwkowski MX. Insights into ErbB signalling from the structure of the ErbB2-pertuzumab complex. Cancer Cell. 2004; 5(4):317-28. [PubMed: 15093539]

7. Olson E. Maximizing human epidermal growth factor receptor 2 inhibition: a new oncologic paradigm in the era of targeted therapy. J Clin Oncol 2012. 2012; 30(14):1712-4. 
8. Agus DB, Akita RW, Fox WD, et al. Targeting ligand-activated ErbB2 signaling inhibits breast and prostate tumor growth. Cancer Cell. 2002; 2(2):127-37. [PubMed: 12204533]

9. Musolino A, Naldi N, Bortesi B, et al. Immunoglobulin $\mathrm{G}$ fragment $\mathrm{C}$ receptor polymorphisms and clinical efficacy of trastuzumab-based therapy in patients with HER-2/neu-positive metastatic breast cancer. J Clin Oncol. 2008; 26(11):1789-96. [PubMed: 18347005]

10. Scheuer W, Friess T, Burtscher H, Bossenmaier B, Endl J, Hasmann M. Strongly enhanced antitumor activity of trastuzumab and pertuzumab combination treatment on HER2-positive human Xenograft tumor models. Cancer Res. 2009; 69(24):9330-6. [PubMed: 19934333]

11. Nahta R, Hung M, Esteva FJ. The HER-2-targeting antibodies trastuzumab and pertuzumab synergistically inhibit the survival of breast cancer cells. Cancer Res. 2004; 64(7):2343-46. [PubMed: 15059883]

12. Adams CW, Allison DE, Flagella K, et al. Humanization of a recombinant monoclonal antibody to produce a therapeutic HER dimerization inhibitor, pertuzumab. Cancer Immunol Immunother. 2006; 55(6):717-27. [PubMed: 16151804]

13. Agus DB, Cordon MS, Taylor C, et al. Phase I clinical study of pertuzumab, a novel HER dimerization inhibitor, in patients with advanced cancer. J Clin Oncol. 2005; 23(11):2534-43. [PubMed: 15699478]

14. Leyland-Jone B. Dose scheduling- herceptin. Oncology. 2001; 61(Suppl 2):31-6. [PubMed: 11694785]

15. Fernando NH, Hurwitz HI. Inhibition of vascular endothelial growth factor in the treatment of colorectal cancer. Semin Oncol. 2003; 30(3 Suppl 6):39-50. [PubMed: 12802794]

16. Yamamoto N, Yamada Y, Yutaka F. Phase I and pharmacokinetic study of HER2-targeted rhuMAb 2C4 (Pertuzumab, RO4368451) in Japanese patients with solid tumors. Jpn J Clin Oncol. 2009; 39(4):260-6. [PubMed: 19261664]

17. Ng CM, Lum BL, Cimenez V, Kelsey S, Allison D. Rationale for fixed dosing of pertuzumab in cancer patients based on population pharmacokinetic analysis. Pharm Res. 2006; 23(6):1275-84. [PubMed: 16715358]

18. Mariani, G.; Strober, W. Immunoglobulin metabolism. In: Metzger, H., editor. Fc Receptors and the Action of Antibodies. American Society for Microbiology; Washington, D.C: 1990. p. 94-177.

19. Henderson LA, Baynes JW, Thorpe SR. Identification of sites of IgG catabolism in the rat. Arch Biochem Biophys. 1982; 215(1):1-11. [PubMed: 7092219]

20. Gianni L, Lladó A, Bianchi G, et al. Open-label, phase II, multicenter, randomized study of the efficacy and safety of two dose levels of pertuzumab, a human epidermal growth factor receptor 2 , dimerization inhibitor, in patients with human epidermal growth factor receptor 2-negative metastatic breast cancer. J Clin Oncol. 2010; 28(7):1131-7. [PubMed: 20124183]

21. Cortés J, Fumoleau P, Bianchi GV, et al. Pertuzumab monotherapy after trastuzumab-based treatment and subsequent reintroduction of trastuzumab: activity and tolerability in patients with advanced human epidermal growth factor receptor 2-positive breast cancer. J Clin Oncol. 2012; 30(14):1594-600. [PubMed: 22393084]

22. Baselga J, Gelmon KA, Verma S, et al. Phase II trial of pertuzumab and trastuzumab in patients with human epidermal growth factor receptor 2-positive metastatic breast cancer that progressed during prior trastuzumab therapy. J Clin Oncol. 2010; 28(7):1738-44.

23. Baselga J, Cortés J, Kim SB, et al. Pertuzumab plus trastuzumab plus docetaxel for metastatic breast cancer. N Engl J Med. 2012; 366(2):109-19. [PubMed: 22149875]

24. Gianni L, Pienkowski T, Im YH, et al. Efficacy and safety of neoadjuvant pertuzumab and trastuzumab in women with locally advanced, inflammatory or early HER2-positive breast cancer (NeoSphere): a randomized mulitcentre, open-label, phase 2 trial. Lancet Oncol. 2012; 13(1):2532. [PubMed: 22153890]

25. Ortega S, Arima A, Chihaya Y, et al. Embryo-Fetal development study of pertuzumab administered by intravenous injection to pregnant cynomolgus monkeys. Int J Toxicol. 2010; 29(1):89-90. [Amer Coll Toxicol 30 ${ }^{\text {th }}$ Annu Meet (Nov 1-4, Palm Springs) 2009]. (Abst P7).

26. Portera C, Walshe J, Rosing D, et al. Cardiac toxicity and efficacy of trastuzumab combined with pertuzumab in patients with trastuzumab-insensitive human epidermal growth factor receptor 2positive metastatic breast cancer. Clin Cancer Res. 2008; 14(9):2710-6. [PubMed: 18451236] 
27. Blackwell K, Burstein H, Storniolo A, et al. Randomized study of lapatinib alone or in combination with trastuzumab in women with ErbB2-positive, trastuzumab-refractory metastatic breast cancer. J Clin Oncol. 2010; 28(7):1124-30. [PubMed: 20124187]

28. Von Minckwitz G, Du Bois A, Schmidt M, et al. Trastuzumab beyond progression in human epidermal growth factor receptor 2-positive advanced breast cancer: a german breast group 26/ breast internal group 03-05 study. J Clin Oncol. 2009; 20(12):1999-2006. [PubMed: 19289619]

29. Cameron D, Casey M, Olivia C, Newstat B, Imwalle B, Geyer CE. Lapatinib plus capecitabine in women with HER-2-positive advanced breast cancer: final survival analysis of a phase III randomized trial. Oncologist. 2010; 15(9):924-34. [PubMed: 20736298]

30. Attard G, Kitzen J, Blagden SP, et al. A phase Ib study of pertuzumab, a recombinant humanized antibody to HER2, and docetaxel in patients with advanced solid tumours. Br J Cancer. 2007; 97(10):1338-43. [PubMed: 18000498]

31. Albanell J, Montagut C, Jones ET, et al. A phase I study of safety and pharmacokinetics of the combination of pertuzumab (rhuMab 2C4) and capecitabine in patients with advanced solid tumors. Clin Cancer Res. 2008; 14(9):2726-31. [PubMed: 18451238]

32. Lu D, Burris HA III, Wang B, et al. Drug interaction potential of trastuzumab emtansine (T-DM1) combined with pertuzumab in patients with HER2-positive metastatic breast cancer. Curr Drug Metab. 2012; 13(7):911-22. [PubMed: 22475266] 


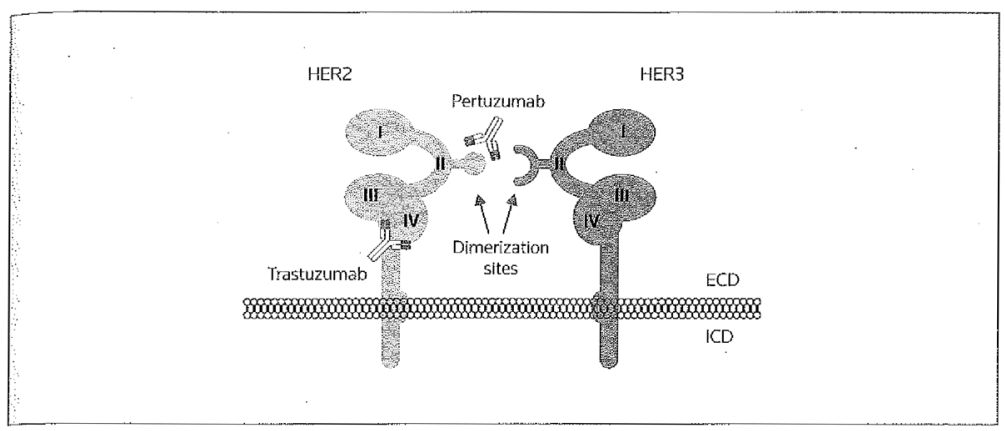

Figure 1.

Heterodimerization of receptor tyrosine-protein kinase erbB-2 (HER2) and erbB-3 (HER3) leads to intracellular downstream signaling that leads to oncologic proliferation. Pertuzumab binds to the dimerization arm (domain II) of HER2 with high affinity, stertically hindering HER2 signaling by inhibiting interactions with HER3. Alternatively, trastuzumab binds to domain IV, inhibiting HER2 function. The combination treatment with pertuzumab and trastuzumab therefore acts as complementary dual blockade of the HER2 receptor, leading to greater efficacy than that with either agent alone. ECD, extracellular domain; ICD, intracellular domain. 


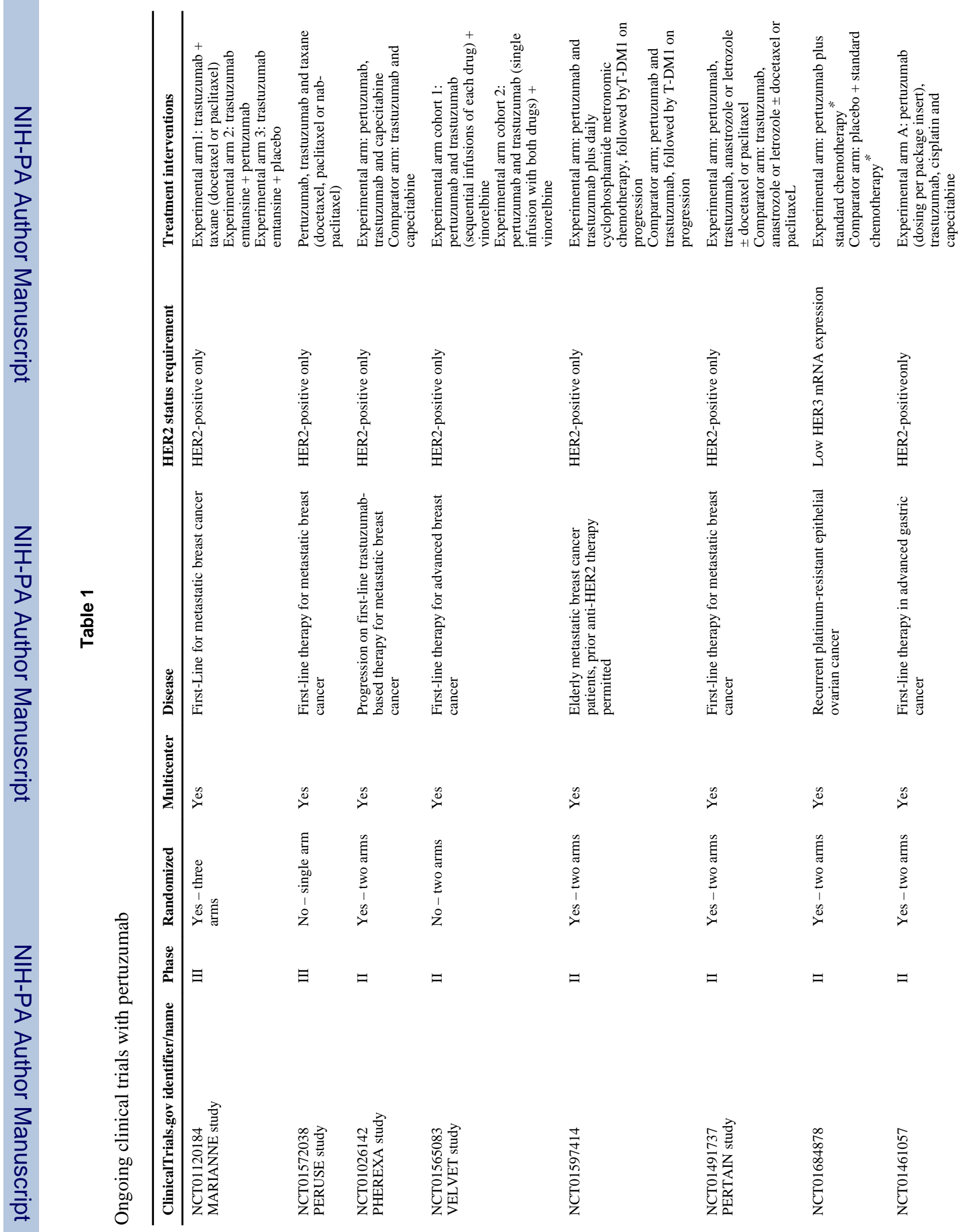


Smith et al.

Page 12

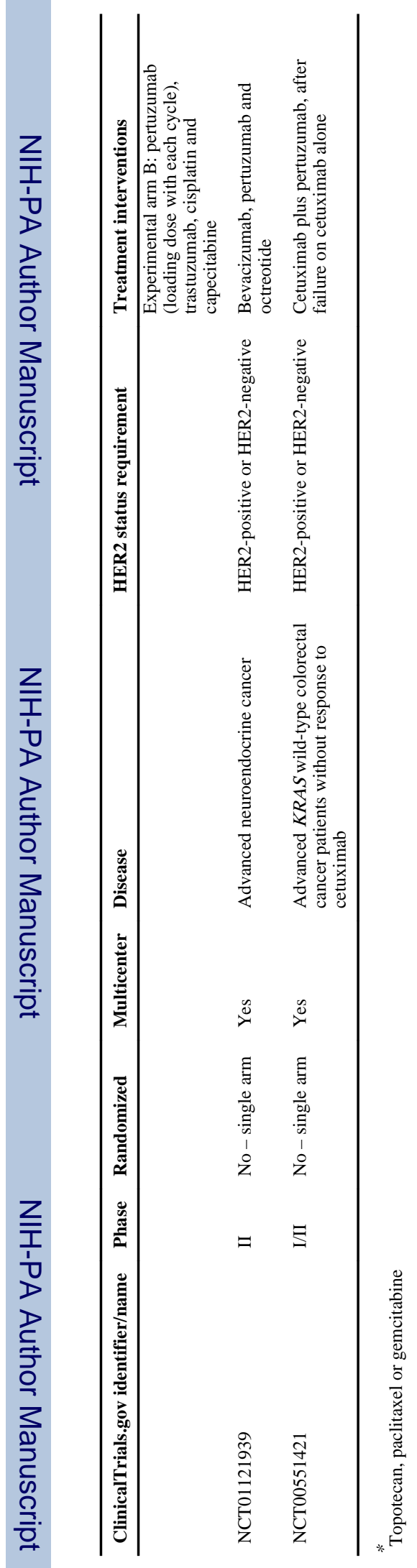

Drugs Today (Barc). Author manuscript; available in PMC 2013 September 30. 